\title{
Selective discrimination of toxic polycyclic aromatic hydrocarbons (PAHs) in water by targeting $\pi$-stacking interactions
}

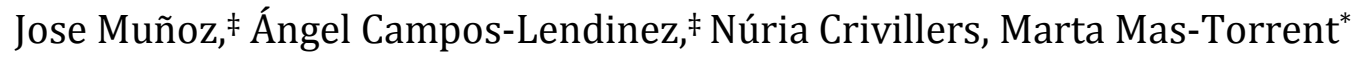 \\ Institut de Ciència de Materials de Barcelona (ICMAB-CSIC) and CIBER-BBN, Campus UAB, 08193 Bellaterra, Spain
}

\begin{abstract}
The development of highly sensitive and selective devices for the rapid screening of polycyclic aromatic hydrocarbons (PAHs) in water is nowadays a crucial challenge owing to their alarming abundance in the environment and adverse health effects. Herein, inspired by the unique $\pi$-stacking interactions taking place between identical small aromatic molecules, a novel, generic and straightforward methodology to electrochemically determine and discriminate such pollutants is described. Such method is focused on covalently anchoring different PAHs on an Indium Tin Oxide (ITO) electrode surface by means of self-assembled monolayers (SAMs). The surface anchored PAHs act as recognition units to selectivity interact with a specific PAH target of the same nature. By tailoring the recognition platform with four different model PAH molecules (naphthalene, anthracene, pyrene and fluoranthene) and carrying out an electronic tongue approximation, the selective discrimination and quantification of the selected PAHs in aqueous samples at ultra-low concentrations was achieved impedimetrically, which was also validated using a certified reference PAH mixture.
\end{abstract}

Keywords: sensors, carbon electrodes, electrochemical impedance spectroscopy, polycyclic aromatic hydrocarbons, supramolecular interactions

\section{INTRODUCTION}

Polycyclic aromatic hydrocarbons (PAHs) are a class of organic pollutants based on fused ring aromatic molecules which possess a potential risk for the environment and human health owing to their carcinogenic and mutagenic properties, as well as high toxicity, persistency and bioaccumulation.1,2,3 More than 100 PAHs are known to be formed naturally, but only 16 of them have been promulgated by different agencies, including the U.S. Environmental Protection Agency (EPA), as priority hazardous compounds. ${ }^{4,5}$ Due to their low water solubility, PAHs are present in the aquatic environment in the form of mixtures at trace levels, being 0.2 ppb the EPA's maximum contaminant level established.

Standard analyses for PAHs quantification are based on using bench-top chromatography instrumentation. ${ }^{6}$ These methods are quite tedious since require lengthy pre-concentration (enrichment), extraction and clean-up steps, which makes them not suitable for their integration into at-point-of-use devices. In this sense, electrochemical techniques open up new alternatives to develop facile and easy to automate analytical approaches since the transduction method is electronic. How- ever, the electrochemical screening of PAHs is nowadays an important analytical challenge due to their trace concentration in water, which implies that the commonly employed voltammetric tools are not sensitive enough to achieve the demanded agency levels.7,8

Surface engineering is a key strategy to achieve highly sensitive electrochemical platforms capable of responding to small external stimuli. ${ }^{9}$ The preparation of self-assembled monolayers (SAMs) is a simple bottom-up route employed to form onto solid supports well-defined and organized monolayers from organic molecules containing free anchoring groups for surface immobilization. ${ }^{10,11,12,13}$ Thus, the preparation of SAMs is highly versatile and has been exploited in a wide range of fields to tune the surface properties,14,15,16,17 including for the development of novel smart electrochemical transducers. ${ }^{18,19,20}$ Very recently, our research group has combined a carbon nanotuberich SAM surface with impedimetric analysis for determining the sum of PAHs in water via $\pi$-stacking interactions, demonstrating promising sensing results. ${ }^{21}$ However, the main limitation of this electronic platform is its inability to discriminate between PAH targets, fact that has been recently achieved utilizing an optical array device. ${ }^{22}$

Herein, inspired by the synergism of the unique supramolecular $\pi$-stacking interactions between identical PAH species ${ }^{23,24}$ with the enhanced sensitivity provided by the SAM-based impedimetric systems, we report the development of a rapid, straightforward and highly sensitive sensor focused on customizing the PAH-based recognition unit for the selective discrimination and quantification of PAH pollutants in aqueous samples. For this goal, Indium Tin Oxide (ITO) electrodes have been covalently tuned with four different PAH-based SAMs as model recognition units (naphthalene (Nap), anthracene (Ant), pyrene (Pyr) and fluoranthene (Flt) to detect PAHs of the same nature. The basis of this work relies on the interesting non-interfering capabilities of an impedimetric device reported previously employing a Pyr molecule as recognition unit towards additional PAH targets. ${ }^{25}$

\section{RESULTS \& DISCUSSION}

Scheme 1 summarizes the synthesis of the different PAHbased recognition platforms via two functionalization steps. Briefly, ITO substrates (ES1) were cleaned in a solvent series with increased polarity (dichloromethane, acetone and ethanol) for 15 min each to degrease. Subsequently, the surfaces were activated in an oxidizing bath $\left(\mathrm{NH}_{4} \mathrm{OH}: \mathrm{H}_{2} \mathrm{O}_{2}: \mathrm{H}_{2} \mathrm{O}, 1: 1: 5\right)$ for $30 \mathrm{~min}$ at $80{ }^{\circ} \mathrm{C}$ to promote the formation of hydroxyl 
groups, rinsed with abundant water and quickly immersed in a dry toluene solution containing 3-glycidoxypropyltrimethoxysilane (GPTMS, 1\% v/v) under inert conditions for $12 \mathrm{~h}$ at 60 ${ }^{\circ} \mathrm{C}$ (step i)), obtaining the ES2 platform. Afterwards, the selected $\mathrm{PAH}$ recognition units bearing an $-\mathrm{NH}_{2}$ group were covalently grafted on the ES2 substrates via epoxy-amine addition. This was realized by immersing them in a $1 \mathrm{mM}$ solution containing either 2-napthylamine, 2-aminoanthracene, 1-aminopyrene or 3-aminofluoranthene $\left(\mathrm{PAH}-\mathrm{NH}_{2}\right)$ in

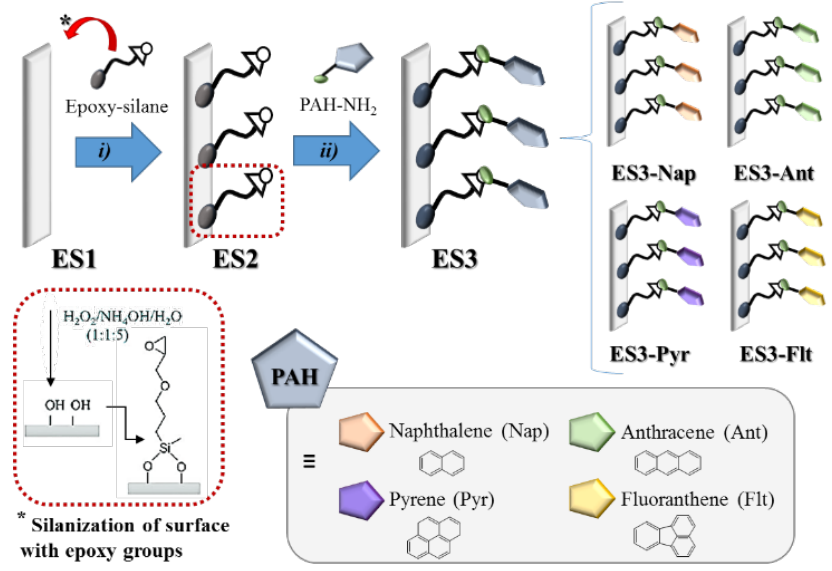

Scheme 1. Schematic representation of the four PAH-based recognition platforms synthesized on ITO electrodes (ES1). i) Electrode activation in an oxidation bath and epoxy-silane SAM formation (ES2). ii) Covalent grafting of the $\mathrm{PAH}-\mathrm{NH}_{2}$ recognition units via epoxy-amine cross-linking (ES3).

toluene at $80{ }^{\circ} \mathrm{C}$ for $24 \mathrm{~h}$ (step ii)). This process resulted in ES3Nap, ES3-Ant, ES3-Pyr and ES3-Flt working electrodes, respectively Importantly, steps (ii) and (iii) were carried out under light exclusion and under inert atmosphere. Substrates were rinsed after each step with plenty of toluene and ethanol in order to remove the unreacted reagents, and finally dried with $\mathrm{N}_{2}$. To optimize the fabrication of the four novel PAHbased systems, the substrates were characterized at each functionalization step by cyclic voltammetry (CV), electrochemical impedance spectroscopy (EIS) and water static contact angle, as is reported in Figure S1-S3 and Table S1.

The feasibility of the different ES3 recognition platforms towards their specific PAH target was studied by EIS (Figure 1). For this aim, the four ES3 substrates were used as working electrodes and dipped into a three-electrode configuration cell filled with a $0.1 \mathrm{M} \mathrm{KCl}$ aqueous solution of $10 \mathrm{mM}\left[\mathrm{Fe}(\mathrm{CN})_{6}\right]^{3-/ 4-}$ as redox marker. The electroanalytical assay is focused on the excellent supramolecular $\pi$-stacking interactions between identical small aromatic structures. ${ }^{23}$ Therefore, concentrations of Nap, Ant, Pyr and Flt analytes in the range 0.5-7.0 ng. $\mathrm{L}^{-}$ 1 were impedimetrically monitored at the ES3-Nap, ES3-Ant, ES3-Pyr and ES3-Flt electrodes, respectively. Aliquots of the different PAHs were directly added into the electrochemical cell and aged for the optimized times of 3, 5, 10 and $8 \mathrm{~min}$, respectively, under soft stirring conditions (see experimental conditions detailed in Supporting Information). The electronic transfer capabilities of each ES3 recognition platform in the frequency domain before and after increasing [PAHs] was determined in terms of charge transfer resistance $\left(\mathrm{R}_{\mathrm{CT}}\right)$. The resulting Nyquist plots (imaginary impedance vs. real impedance) were fitted with a typical Randles equivalent circuit. ${ }^{9}$
Figure 1 A-D evidenced an interesting $R_{C T}$ linear decrease trend with increasing the [PAHs]. From an electrochemical point of view, a positive impedimetric change would be expected after supramolecular binding formation because an insulating layer on the electrode surface is generated. However, this reverse behavior can be accounted by a SAM reorganization after PAH-PAH interactions. This fact induces the formation of empty channels exposed on the electrode surface, favoring the penetration of the $\left[\mathrm{Fe}(\mathrm{CN})_{6}\right]^{3-/ 4-}$ redox marker towards the electrode surface and therefore, facilitating its redox reaction. ${ }^{25} \mathrm{~A}$ blank reference test was run at the bare ES1 electrode using Pyr as a model PAH analyte. As shown in Figure S4, no significant impedimetric changes were observed even employing concentrations $10^{3}$ times larger, demonstrating the leading

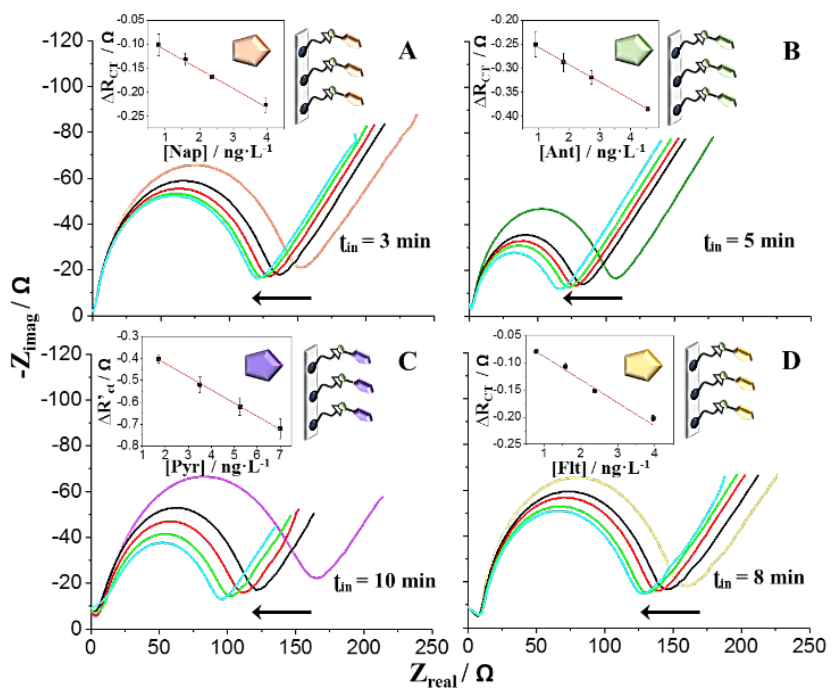

Figure 1. Nyquist plots corresponding to the impedance sensing at the A) ES3-Nap, B) ES3-Ant, C) ES3-Pyr and D) ES3-Flt recognition platforms before and after interacting with their corresponding PAH target at different concentrations (tin: incubation time). Inset: calibration plot with their corresponding error bars $(n=9)$. EIS measurements obtained utilizing a $0.1 \mathrm{M}$ $\mathrm{KCl}$ solution containing $10 \mathrm{mM}\left[\mathrm{Fe}(\mathrm{CN})_{6}\right]^{3-/ 4-}$ as redox marker.

role of the PAH-based SAM recognition unit on the supramolecular complex formation.

For the calibration curve construction (see Figure 1, insets), EIS experiments were carried out employing three different electrodes from two different batches in order to explore the reproducibility of the experimental procedure and each measurement was taken per triplicate in order to determine the repeatability of the analytical signal $(n=9)$. Importantly, it was tested that the sensing platform i.e., the electrodes, could be stored and used two weeks after their fabrication without altering the performance. The low error bars from the calibration curves in Figure 1 demonstrates that the method is reproducible and repeatable, and the devices are stable along the time. The relative $\Delta \mathrm{R}^{\prime}$ Ст signal was calculated as follows:

$\Delta \mathrm{R}_{\mathrm{CT}}^{\prime}=\Delta \mathrm{R}_{\mathrm{CT}} / \mathrm{R}_{o}$

(Equation 1)

$\Delta \mathrm{R}_{\mathrm{CT}}=\mathrm{R}_{i}-\mathrm{R}_{o}$

(Equation 2)

where $\mathrm{R}$ corresponds to the electron transfer resistance of the electrodes before $\left(\mathrm{R}_{o}\right)$ and after $\left(\mathrm{R}_{i}\right)$ incubation with the differ- 
ent PAH concentrations. Importantly, all four ES3 systems exhibited excellent regression curves $\left(\mathrm{r}^{2}>0.99\right)$ with good sensitivities (i.e., slope) as well as extremely low detection limits (LOD) for their corresponding PAH target in the order of part per trillion (ppt) levels, as summarized in Table 1. Therefore, the synergism of combining the high sensitivity and fast response EIS technique with a SAM-based active sensing layer, permits to simply translate any slight surface modification (as the reported supramolecular $\pi$-stacking interactions between small aromatic molecules at trace levels) into a detectable output signal.

Since anti-interference property is a critical factor for sensors, the following step was focused on interrogating the selectivity of the developed ES3 recognition electrodes towards their corresponding PAH target (Figure 2). Accordingly, effects of potential co-existing PAHs were impedimetrically examined for each ES3 system by analyzing separately five different PAH species, such as Nap, Ant, Pyr, Flt, and coronene (Cor), with 2, $3,4,4$ and 7 aromatic rings, respectively $\left([\mathrm{PAH}]_{\mathrm{x}}=2 \mathrm{ppt}\right)$. As depicted in Figure 2 (dashed line), each ES3 system is much more sensitive and, therefore, selective, to the PAH target equal to its recognition group, with interfering signals mostly lower than $10 \%$ in the same concentration

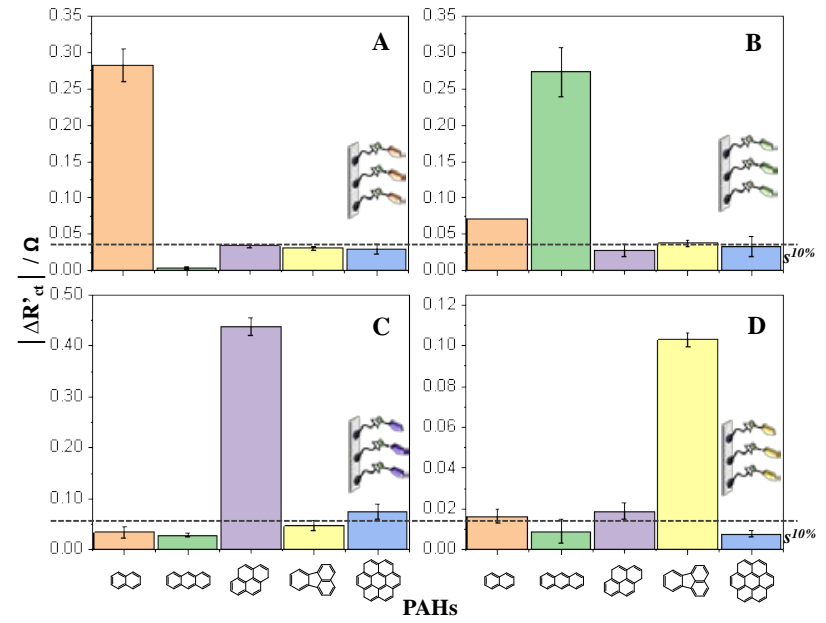

Figure 2. Electrochemical anti-interfering assay at the A) ES3Nap, B) ES3-Ant, C) ES3-Pyr and D) ES3-Flt using five different PAH analytes (Nap, Ant, Pyr, Flt and Cor) with a 2 ppt concentration (dashed line: $10 \%$ of the signal derived from its corresponding PAH target, $\mathrm{s}^{10 \%}$ ). Bar charts are represented with their corresponding error bars $(n=9)$. Redox marker: $10 \mathrm{mM}$ $\left[\mathrm{Fe}(\mathrm{CN})_{6}\right]^{3-/ 4-}$.

range. These results reflect advanced supramolecular $\pi$-stacking interactions between molecules exhibiting the same aromatic skeleton (see also Figure S5) over other PAH components which might be present in similar concentrations, driving the superior selectivity of the reported electrodes. Considering the selected PAHs studied, it can be affirmed that such selectivity does not arise only from the number of aromatic rings of the PAHs but instead relies on their molecular structure. This is reflected with the cases of Pyr and Flt; although both systems have the same number of aromatic rings, they are not significantly interfering with each other.

Further, an electronic tongue approximation was also carried out in order to evaluate the applicability of the developed method in a complex mixture solution. ${ }^{26}$ Figure 3A shows schematically the followed procedure employing the ES3-Nap as a model PAH-based recognition platform, which consists in the following steps: the ES3-Nap substrate was impedimetrically measured in the three-electrode configuration cell $a$ ) before and $b$ ) after adding 2 ppt of Nap (its intrinsic PAH target). Afterwards, $c$ ) the three additional PAHs (Ant, Flt and Pyr for this model system) were added and aged into the electrochemical cell to study the interference signal in terms of $\mathrm{R}_{\mathrm{CT}}$ changes. Finally, $d)$ an additional 2 ppt of a Nap aliquot ([Nap $\left.]_{\mathrm{T}}=4 \mathrm{ppt}\right)$ was spiked for exploring the sensing discrimination in a complex matrix media. The same methodology was carried out for the other three ES3 recognition platforms (ES3-Ant, ES3-Pyr and ES3-Flt), and the data is presented in Table S2.

Table 1. Electrode response parameters extracted from the calibration curves with their corresponding confident intervals $(n=9)$.

\begin{tabular}{|l|l|l|l|l|}
\hline ES3 & Analyte & $\mathrm{r}^{2}$ & $\begin{array}{l}\text { Sensitivity } \\
\left(\Omega \cdot \mathrm{L} \cdot \mathrm{ng}^{-1}\right)\end{array}$ & $\begin{array}{l}\mathrm{LOD} \\
\left(\mathrm{ng} \cdot \mathrm{L}^{-1}\right)\end{array}$ \\
\hline ES3-Nap & Nap & 0.993 & $\begin{array}{l}-0.040 \pm \\
0.002\end{array}$ & $0.79 \pm 0.08$ \\
\hline ES3-Ant & Ant & 0.999 & $\begin{array}{l}-0.036 \pm \\
0.004\end{array}$ & $0.91 \pm 0.09$ \\
\hline ES3-Pyr & Pyr & 0.999 & $\begin{array}{l}-0.059 \pm \\
0.002\end{array}$ & $1.70 \pm 0.06$ \\
\hline ES3-Flt & Flt & 0.993 & $\begin{array}{l}-0.043 \pm \\
0.001\end{array}$ & $0.79 \pm 0.01$ \\
\hline
\end{tabular}
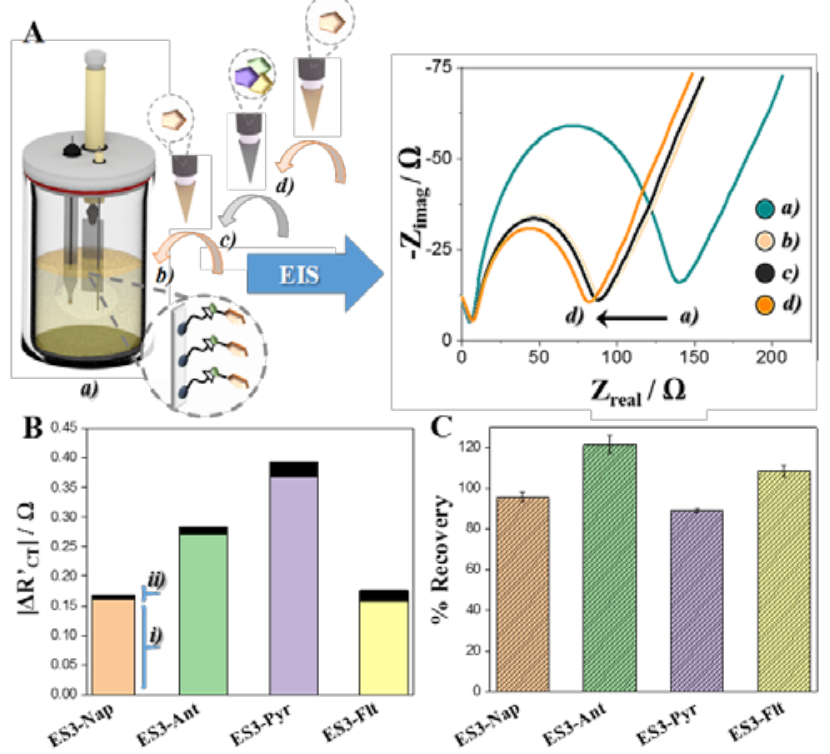

Figure 3. A) Exemplification of the electronic tongue approximation carried out into the three-electrode configuration cell using the ES3-Nap as the model recognition platform, where the signal was measured: $a$ ) before and $b$ ) after adding a fixed PAH target concentration ( $\left.\left.[\mathrm{Nap}]=2 \mathrm{ppt} ;[\mathrm{PAH}]_{\mathrm{T}}=2 \mathrm{ppt}\right), c\right)$ after adding a same fixed concentration of the interferent PAHs $\left([\right.$ Ant $\left.]=[\mathrm{Pyr}]=[\mathrm{Flt}]=2 \mathrm{ppt} ;[\mathrm{PAH}]_{\mathrm{T}}=8 \mathrm{ppt}\right)$ and $\left.d\right)$ after adding 
again a fix concentration of the $\mathrm{PAH}$ target $([\mathrm{Nap}]=2 \mathrm{ppt}$; $[\mathrm{Nap}]_{\mathrm{T}}=4 \mathrm{ppt}$ and, therefore, $[\mathrm{PAH}]_{\mathrm{T}}=10 \mathrm{ppt}$ ). B) Relative $\left|\Delta \mathrm{R}_{\mathrm{CT}}^{\prime}\right|$ signals derived from a $[\mathrm{PAH}]_{\text {target }}=2 \mathrm{ppt} i$ ) before and ii) after adding the three additional PAHs (interfering study). C) \%Recovery yielded for $[\mathrm{PAH}]_{\text {target }}=4 \mathrm{ppt}$ in the complex matrix. Redox marker: $10 \mathrm{mM}\left[\mathrm{Fe}(\mathrm{CN})_{6}\right]^{3-/ 4-}$.

Bar charts from Figure 3B represent the relative $\left|\Delta R^{\prime}{ }_{C T}\right|$ signal for a $[\mathrm{PAH}]_{\operatorname{target}}=2 \mathrm{ppt} i$ ) before and ii) after adding $2 \mathrm{ppt}$ of each additional interfering $\mathrm{PAH}$, with a final concentration of $[\mathrm{PAH}]_{\mathrm{T}}=8 \mathrm{ppt}$. It can be observed that there is not a significant increase in the impedimetric signal after the three additional PAHs are added into the electrochemical cell, with a $\left|\Delta \mathrm{R}_{\mathrm{CT}}\right|$ increase of $4.3 \%, 4.4 \%, 7.7 \%$ and $7.1 \%$ for ES3-Nap, ES3-Ant, ES3-Pyr and ES3-Flt, respectively. Therefore, this demonstrates that the electroanalytical response of the PAH-based recognition platforms is specific to their $\mathrm{PAH}$ target. Additionally, Figure 3C shows the \%recovery achieved for a [PAH] target $=4 \operatorname{ppt}(\operatorname{after}$ step $d)$ ), and therefore, in the complex matrix media with $[\mathrm{PAH}]_{\mathrm{T}}=10 \mathrm{ppt}$. The calibration curves from Figure 1 were used for each interpolation. Overall, it is important to point out the excellent recovery achieved in complex matrices after interpolating the average impedimetric response on the calibration curves for $[\mathrm{PAH}]_{\text {target }}=4 \mathrm{ppt}$ (following the data reported in Table S2, 95.7\%, 118\%, 89.0\% and 108\% for ES3Nap, ES3-Ant, ES3-Pyr and ES3-Flt, respectively). Thus, these promising results demonstrate again that the interaction between identical PAH species is the most significant among all the PAHs tested in this work even in complex matrices. In this sense, it is possible to tune the sensibility of the developed surfaces towards selected PAHs by tailoring the PAH-based recognition unit.

Finally, the non-interfering method was additionally explored by employing a certified reference material (EPA 525 PAH kit from Sigma-Aldrich, which contains 13 different PAHs, including Ant and Pyr but excluding Nap and Flt, which would act here as the negative blanks (see Figure $\mathbf{4}$ for schematic illustration) for the validation of the calibration sensing curves. After spiking the electrolyte redox marker solution with the certified standard solution

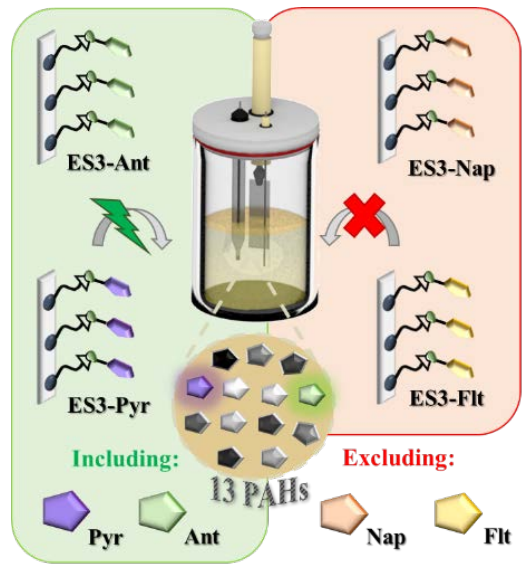

Figure 4. Schematic illustration of the non-interfering assay involving a certified reference material containing 13 different PAHs $\left([\mathrm{PAH}]_{\mathrm{T}}=26 \mathrm{ppt}\right)$, which includes Ant and Pyr ([Ant $]=$ [Pyr] = 2 ppt, being impedimetrically recognized by the ES3Ant and ES3-Pyr, respectively) and excludes Nap and Flt
([Nap $]=[\mathrm{Flt}]=0 \mathrm{ppt}$, negative blanks and therefore, no impedimetric responses are obtained employing the ES3-Nap and ES3-Flt). Redox marker: $10 \mathrm{mM}\left[\mathrm{Fe}(\mathrm{CN})_{6}\right]^{3-/ 4-}$.

containing 2 ppt of each PAH $\left([\mathrm{PAH}]_{\mathrm{T}}=26 \mathrm{ppt}\right.$, with $[\mathrm{Ant}]=$ $[\mathrm{Pyr}]=2 \mathrm{ppt}$ and $[\mathrm{Nap}]=[\mathrm{Flt}]=0 \mathrm{ppt})$, good recoveries were achieved for the ES3-Ant (87\%) and ES3-Pyr (101\%) after interpolating the average impedimetric response $(n=3)$ on their corresponding calibration curves (see Figure S6 and Table S3). Remarkably, the ES3-Nap and ES3-Flt electrodes (negative blank experiments) did not present significant responses (13\% and $2 \%$, respectively). Hence, it has been elucidated that the here-reported engineered sensing surface is reproducible, robust and versatile since it can be modified for switching the selectivity of the transducer platform towards the selected EPA's PAH pollutant target.

\section{CONCLUSIONS}

In summary, the electrochemical discrimination of hazardous $\mathrm{PAH}$ pollutants in aqueous samples has been successfully achieved by taking advantage of the unique supramolecular $\pi-$ stacking interactions that take place between identical aromatic small molecules. The developed method, which could be easily miniaturized since the transduction principle is electronic, paves the way for customizing electrodes with the desired recognition unit for selectively screening a broad range of small aromatic molecules.

\section{MATERIALS AND METHODS}

Chemical and Reagents. ITO substrates (single-face coated ITO over unpolished float glass; resistance: 15-25 $\Omega$ ) were purchased from Delta Technologies. Both SAM-based reagents (glycidoxypropyl-trimethoxysilane ( $\geq 98 \%$ ), 1-aminopyrene (97\%), 2-aminoanthracene (96\%), 1-naphthylamine ( $\geq 99 \%)$ and 3 -aminofluoranthene (90\%)) and PAH targets (pyrene $(\geq$ 99\%), anthracene (99\%), naphthalene (99\%), fluoranthene (98\%) and coronene (97\%)) were purchased from Sigma-Aldrich. Potassium chloride ( $\mathrm{KCl}, 99.5 \%)$, potassium ferricyanide/ferrocyanide $\left(\mathrm{K}_{3}\left[\mathrm{Fe}(\mathrm{CN})_{6}\right] / \mathrm{K}_{4}\left[\mathrm{Fe}(\mathrm{CN})_{6}\right]\right.$, 99.8\%), ammonium hydroxide $\left(\mathrm{NH}_{4} \mathrm{OH}, 5 \mathrm{~N}\right)$ and hydrogen peroxide $\left(\mathrm{H}_{2} \mathrm{O}_{2}\right.$, $30 \%$ wt.) were also supplied by Sigma-Aldrich. All the organic solvents (dichloromethane, acetone, ethanol and toluene) used were HPLC grade and acquired by Teknokroma. Aqueous solutions were prepared employing ultra-pure water from a Milli$\mathrm{Q}$ system (Millipore).

Apparatus. Electrochemical experiments were carried out employing a Novocontrol Alpha-AN impedance analyzed with a potentiostat POT/GAL 30V/2A electrochemical interface. Both cyclic voltammetry (CV) and electrochemical impedance spectroscopy (EIS) experiments were run in a three-electrode configuration cell filled with a $20 \mathrm{~mL} 0.1 \mathrm{M} \mathrm{KCl}$ solution containing $0.01 \mathrm{M} \mathrm{K}_{3}\left[\mathrm{Fe}(\mathrm{CN})_{6} / \mathrm{K}_{4}\left[\mathrm{Fe}(\mathrm{CN})_{6}\right]\right.$. The electrodes employed were: a single junction $\mathrm{Ag} / \mathrm{AgCl}$ as the refence electrode, a platinum wire as the counter electrode and the ITO-based substrates as the working electrodes). The scan rate employed for $\mathrm{CV}$ measurements was $50 \mathrm{mV} \cdot \mathrm{s}^{-1}$. EIS measurements were acquired in a frequency range from $100 \mathrm{kHz}$ to $0.1 \mathrm{~Hz}$, using a bias of $+150 \mathrm{mV}$ and an AC amplitude of $5 \mathrm{mV}$. Charge transfer resistance ( $\left.R_{c T}\right)$ parameters were acquired by fitting the impedance spectra to the Randles equivalent circuit with Zview software (Scribner Associates Inc.). 
The contact angle $(\theta)$ measurements were obtained from a DSA 100 System (KRÜSS) by automatically dispensing $3 \mu \mathrm{L}$ Milli-Q water drops. Experiments were obtained per triplicate $(n=3)$.

Electroanalytical assay. The electroanalytical assay was impedimetrically monitorized by means of $\mathrm{R}_{\mathrm{CT}}$ changes. The different ES3 working electrodes were immersed in an aqueous solution containing a desired concentration of the selected PAH for some minutes. Such desired concentrations were achieved by adding aliquots from the stock PAH solutions: naphthalene $32 \mathrm{ppm}$, anthracene $7.3 \mathrm{ppb}$, fluoranthene $26 \mathrm{ppb}$, pyrene 135 ppb and coronene $14 \mathrm{ppb}$. Each measurement was token per triplicate in three different electrodes from two different batches $(n=9)$ in order to evaluate both repeatability and reproducibility. Electrodes were used during two different weeks and exhibited good stability in that time.

\section{ASSOCIATED CONTENT}

\section{Supporting Information}

Extended experimental procedures, physical and electrochemical characterizations, blank experiments and electroanalytical data derived from the certified reference material are included in the supporting information. This material is available free of charge via the internet http://pubs.acs.org.

\section{AUTHOR INFORMATION}

\section{Corresponding Author}

Dr. Marta Mas-Torrent, e-mail:mmas@icmab.es.

\section{Author Contributions}

‡These authors contributed equally.

\section{Notes}

The authors declare no competing financial interests.

\section{ACKNOWLEDGMENTS}

This work was funded by the ERC StG 2012306826 e-GAMES. The authors also thank the Networking Research Center on Bioengineering, Biomaterials, and Nanomedicine (CIBER-BBN), the DGI (Spain) project FANCY CTQ2016-80030-R, the Generalitat de Catalunya (2017-SGR-918) and the Spanish Ministry of Economy and Competitiveness, through the "Severo Ochoa" program for Centers of Excellence in R\&D (SEV-2015-0496). Dr. J. M. gratefully acknowledges the "Juan de la Cierva" program. A. C.-L. is enrolled in the UAB PhD program and acknowledges a FPI fellowship.

\section{REFERENCES}

(1) White, A. J.; Bradshaw, P. T.; Herring, A. H.; Teitelbaum, S. L.; Beyea, J.; Stellman, S. D.; Steck, S. E.; Mordukhovich, I.; Eng, S. M.; Engel, L. S. Exposure to Multiple Sources of Polycyclic Aromatic Hydrocarbons and Breast Cancer Incidence. Environ. Int. 2016, 89, 185-192.

(2) Shen, H.; Huang, Y.; Wang, R.; Zhu, D.; Li, W.; Shen, G.; Wang, B.; Zhang, Y.; Chen, Y.; Lu, Y. Global Atmospheric Emissions of Polycyclic Aromatic Hydrocarbons from 1960 to 2008 and Future Predictions. Envrion. Sci. Technol. 2013, 47, 6415-6424.

(3) Manoli, E.; Kouras, A.; Karagkiozidou, O.; Argyropoulos, G.; Voutsa, D.; Samara, C. Polycyclic Aromatic Hydrocarbons
(PAHs) at Traffic and Urban Background Sites of Northern Greece: Source Apportionment of Ambient PAH Levels and PAH-induced Lung Cancer Risk. Environ. Sci. Pollut. R. 2016, 23, 3556-3568.

(4) Bruzzoniti, M. C.; Fungi, M.; Sarzanini, C. Determination of EPA's Priority Pollutant Polycyclic Aromatic Hydrocarbons in Drinking Waters by Solid Phase Extraction-HPLC. Anal. Methods 2010, 2, 739-745.

(5) Keith, L. H. The Source of US EPA's Sixteen PAH Priority Pollutants. Polycycl. Aromat. Comp. 2015, 35, 147-160.

(6) Bansal, V.; Kumar, P.; Kwon, E. E.; Kim, K.-H. Review of the Quantification Techniques for Polycyclic Aromatic Hydrocarbons (PAHs) in Food Products. Cr. Rev. Food Sci. 2017, 57, 3297-3312.

(7) Díaz-González, M.; Gutiérrez-Capitán, M.; Niu, P.; Baldi, A.; Jiménez-Jorquera, C.; Fernández-Sánchez, C. Electrochemical Devices for the Detection of Priority Pollutants Listed in the EU Water Framework Directive. TrAC, Trends Anal. Chem. 2016, 77, 186-202.

(8) Nsibande, S.; Montaseri, H.; Forbes, P. Advances in the Application of Nanomaterial-based Sensors for Detection of Polycyclic Aromatic Hydrocarbons in Aquatic Systems. $\operatorname{Tr} A C$, Trends Anal. Chem. 2019, 115, 52-69.

(9) Muñoz, J.; Montes, R.; Baeza, M. Trends in Electrochemical Impedance Spectroscopy Involving Nanocomposite Transducers: Characterization, Architecture Surface and BioSensing. TrAC, Trends Anal. Chem. 2017, 97, 201-215.

(10) Marchante, E.; Crivillers, N.; Buhl, M.; Veciana, J.; MasTorrent, M. An Electrically Driven and Readable Molecular Monolayer Switch Based on a Solid Electrolyte. Angew. Chem. Int. Ed. 2016, 128, 376-380.

(11) Mas-Torrent, M.; Rovira, C.; Veciana, J. Surface-Confined Electroactive Molecules for Multistate Charge Storage Information. Adv. Mat. 2013, 25, 462-468.

(12) Gupta, T.; van der Boom, M. E. Redox-Active Monolayers as a Versatile Platform for Integrating Boolean Logic Gates. Angew. Chem. Int. Ed. 2008, 47, 5322-5326.

(13) Casalini, S.; Bortolotti, C. A.; Leonardi, F.; Biscarini, F. Self-Assembled Monolayers in Organic Electronics. Chem. Soc. Rev. 2017, 46, 40-71.

(14) Simão, C.; Mas-Torrent, M.; Veciana, J.; Rovira, C. Multichannel Molecular Switch with a Surface-confined Electroactive Radical Exhibiting Tunable Wetting Properties. Nano Lett. 2011, 11, 4382-4385.

(15) de Boer, B.; Hadipour, A.; Mandoc, M. M.; van Woudenbergh, T.; Blom, P. W. Tuning of Metal Work Functions with Self-Assembled Monolayers. Adv. Mat. 2005, 17, 621-625.

(16) Angelerou, M. G. F.; Sabri, A.; Creasey, R.; Angelerou, P.; Marlow, M.; Zelzer, M. Surface-directed Modulation of Supramolecular Gel Properties. Chem. Comm. 2016, 52, 42984300.

(17) Galanti, A.; Diez-Cabanes, V.; Santoro, J.; Valášek, M.; Minoia, A.; Mayor, M.; Cornil, J. r. m.; Samorì, P. Electronic Decoupling in C3-symmetrical Light-responsive Tris (azobenzene) Scaffolds: Self-Assembly and Multiphotochromism. J. Am. Chem. Soc. 2018, 140, 1606216070.

(18) Mandler, D. Chiral Self-Assembled Monolayers in Electrochemistry. Curr. Opin. Electrochem. 2018, 7, 42-47.

(19) Wong, R. A.; Yokota, Y.; Wakisaka, M.; Inukai, J.; Kim, Y. Discerning the Redox-Dependent Electronic and Interfacial Structures in Electroactive Self-Assembled Monolayers. J. Am. Chem. Soc. 2018, 140, 13672-13679. 
(20) Paul, K. B.; Kumar, S.; Tripathy, S.; Vanjari, S. R. K.; Singh, V.; Singh, S. G. A Highly Sensitive Self-Assembled Monolayer Modified Copper Doped Zinc Oxide Nanofiber Interface for Detection of Plasmodium Falciparum Histidine-rich Protein-2: Targeted Towards Rapid, Early Diagnosis of Malaria. Biosens. Bioelectron. 2016, 80, 39-46.

(21) Muñoz, J.; Navarro-Senent, C.; Crivillers, N.; Mas-Torrent, M. Study of Carbon Nanotube-rich Impedimetric Recognition Electrode for Ultra-Low Determination of Polycyclic Aromatic Hydrocarbons in Water. Microchim. Acta 2018, 185, 255-.

(22) Tropp, J.; Ihde, M. H.; Williams, A. K.; White, N. J.; Eedugurala, N.; Bell, N. C.; Azoulay, J. D.; Bonizzoni, M. A Sensor Array for the Discrimination of Polycyclic Aromatic Hydrocarbons Using Conjugated Polymers and the Inner Filter Effect. Chem. Sci. 2019, 10, 10247-10255.

(23) Liu, S.; Wei, M.; Zheng, X.; Xu, S.; Zhou, C. Highly Sensitive and Selective Sensing Platform based on $\pi-\pi$ Interaction between Tricyclic Aromatic Hydrocarbons with ThionineGraphene Composite. Anal. Chim. Acta 2014, 826, 21-27.

(24) Shukla, A. D.; Strawser, D.; Lucassen, A. C.; Freeman, D.; Cohen, H.; Jose, D. A.; Das, A.; Evmenenko, G.; Dutta, P.; Van Der Boom, M. E. Covalent assembly of stilbene-based monolayers: Factors controlling molecular interactions. J. Phis. Chem. B 2004, 108, 17505-17511.

(25) Muñoz, J.; Crivillers, N.; Mas-Torrent, M. Carbon-Rich Monolayers on ITO as Highly Sensitive Platforms for Detecting Polycyclic Aromatic Hydrocarbons in Water: The Case of Pyrene. Chemistry-A European Journal 2017, 23, 15289-15293.

(26) Podrażka, M.; Bączyńska, E.; Kundys, M.; Jeleń, P. S.; Witkowska Nery, E. Electronic Tongue - A Tool for All Tastes? Biosensors 2018, 8, 3. 